\title{
Título Superior De Música. Relación Entre La Motivación De Logro Musical Y Las Conductas Desadaptativas De Abandono En Estudiantes
}

\author{
Francisco Javier Zarza-Alzugaray, Dr. \\ Universidad Complutense de Madrid, Spain
}

Doi: 10.19044/esj.2018.v14n10p46 URL:http://dx.doi.org/10.19044/esj.2018.v14n10p46

\begin{abstract}
The motivation of musical achievement is one of the main requirements to achieve academic success during the formative period of the students (Zarza, 2014). In this sense, behaviors such as the consumption of substances or cognitions such as thoughts of dropping out of musical studies are usually very present in music students and generate long-term maladaptive behavior sustained over time that can prevent a correct musical training or even the actual abandonment of them. We present a first approach to the relationship between motivation and substance use, as well as the presence of thoughts of abandonment. In this sense, an improvement is proposed in the training plans that tend to a greater individualization and surveillance of the presence of this type of behavior linked with low motivational levels.
\end{abstract}

Keywords: Music education, music students, sensitivity to reward, sensitivity to punishment, achieve motivation

\section{Resumen}

La motivación de logro musical es uno de los principales requerimientos para conseguir, durante el periodo formativo de los estudiantes, éxito académico (Zarza, 2014). En este sentido, conductas como el consumo de sustancias o cogniciones como los pensamientos de abandono de los estudios musicales suelen estar muy presentes en los estudiantes de música; asimismo, generan a largo plazo comportamientos desadaptativos sostenidos en el tiempo que pueden llegar a impedir una correcta formación musical o incluso el abandono real de los mismos. Se presenta una primera aproximación al estudio de la relación entre la motivación con el consumo de sustancias, así como con la presencia de pensamientos de abandono en estudiantes de música. En consecuencia, se propone una mejora en los planes formativos que tiendan a una mayor individualización y vigilancia de la presencia de este tipo de conductas ligadas con bajos niveles motivacionales. 
Palabras clave: educación musical, estudiantes de música, motivación de logro musical, consumo de sustancias, pensamientos de abandono.

\section{Introducción}

Desde algunas décadas, se tiene asumido por la comunidad científica que la motivación de logro es un constructo en el que confluyen distintas teorías interpretativas del mismo, y han sido numerosas las investigaciones que han relacionado diversos constructos antaño separados (Zarza, 2014).

La conducta que se exhibe para la consecución de un determinado logro, está caracterizada por presentar una direccionalidad en tanto en cuanto conlleva una elección personal de las acciones a realizar y una persistencia en el mantenimiento en el tiempo de dicha conducta. Por su parte, son importantes en la génesis de conductas encaminadas hacia la consecución del logro, diferencias individuales como la ansiedad ante el éxito o el fracaso, en el logro de la tarea (Manassero y Vázquez, 1998).

El modelo de Weiner (1986) facilita la comprensión del estilo atributivo, el cual presupone que ante la ocurrencia de un evento, se le busca explicaciones que en ocasiones no coinciden con la realidad; pero, que sin embargo, inciden en los sentimientos que se generan y en las conductas posteriores, de ahí que se entienda que presentan un valor motivacional en una conducta determinada.

Este modelo, desde una perspectiva integradora y atribucional, asume que si el resultado es inesperado, negativo o importante, el sujeto buscará la causa del resultado teniendo en cuenta los antecedentes de información con los que se contaba, historia personal pasada, historia de otros respecto de dicha tarea, las reglas causales, la perspectiva del actor y del observador, los sesgos atribucionales etc. Así, desencadenarán en la atribución del resultado a una serie de causas particulares, tales como la capacidad, el esfuerzo realizado para la obtención de la meta, las demandas específicas de la tarea, la suerte, las características físicas y personales del individuo, etc. (Zarza, 2014).

A partir del modelo de Weiner se puede asumir que el proceso de motivación puede ser descrito en términos de cuatro grandes dimensiones: cogniciones adaptativas, comportamiento adaptativo, cogniciones impeditivas o maladaptativas y comportamiento maladaptativo (Martin, 2001, 2003, 2007); y se debe tener en cuenta que la conciencia de estar envueltos en determinadas acciones puede ser motivo constituyente del sentido de eficacia de los comportamientos que se estén llevando a cabo, así como de la habilidad de la propia persona para atribuir el origen y las consecuencias de las acciones que se realizan (David, Newen y Vogeley, 2008; Gallagher, 2000, 2008).

Las cogniciones adaptativas vienen representadas por constructos como la autoeficacia, la valoración del logro y la adquisición de dominio en la 
tarea. Las cogniciones impeditivas o maladaptativas vienen determinadas por constructos como la evitación del fallo, sensación de falta de control sobre la situación y ansiedad. El comportamiento adaptativo viene descrito por la planificación correcta, el dominio de la tarea y la persistencia, mientras que el comportamiento maladaptativo está caracterizado por el auto-hándicap y la falta de compromiso con la resolución de la tarea (Martin, 2008) y puede acarrear consecuencias como el abandono en este caso de los estudios musicales o el consumo de sustancias para el afrontamiento de esas situaciones percibidas con poco control.

El compromiso con los mismos merece especial atención y enlaza con el estudio de la motivación para la consecución de logros, entendido éste como la obligación contraída para con la resolución de la tarea, bien haya sido ésta encomendada por otros o asumida por uno mismo. Así pues, encontramos que en diferentes ámbitos como el académico, el deportivo o el musical, está claro que hay características principales y atributos relevantes que pueden predecir las actuaciones futuras; en este sentido, por ejemplo, tanto la motivación como el compromiso con la tarea son causas determinantes en el logro de objetivos y metas (Martin, 2008). En términos de las teorías del locus de control, se ha encontrado que cuando los músicos perciben más control y autonomía en la situación existe una mayor perseverancia en el afrontamiento de las situaciones difíciles (Renwick y McPherson, 2002).

En el sentido de todo lo expuesto hasta aquí, el objetivo de esta investigación no es otro que el de comprobar si los distintos niveles motivacionales de los estudiantes están ligados a la presencia de conductas desadaptativas como los pensamientos de abandono de los estudios musicales o el consumo de sustancias ante las actuaciones públicas.

\section{Método}

\section{Participantes}

La muestra está compuesta por 154 estudiantes (83 hombres y 71 mujeres y un caso perdido) del Título Superior de Música pertenecientes a 5 centros de formación superior españoles. La edad media de los estudiantes es de 21.80 (D.T. $=3.80$ ) años con un rango desde los 16 hasta los 44 años.

En cuanto a las familias instrumentales de pertenencia encontramos 44 instrumentistas de viento madera, 24 de viento metal, 48 de cuerda frotada, 18 instrumentistas de tecla, 10 de cuerda pulsada, 5 percusionistas y 5 estudiantes de canto.

\section{Procedimiento}

Tras una primera toma de contacto con la dirección de los centros superiores de música que solicitaron participar en el estudio, un miembro del equipo de investigación se desplazó hasta ellos para hacer un mejor acopio del 
cuestionario diseñado ad hoc y que debía ser respondido por los estudiantes de los centros.

\section{Instrumentos y análisis de datos}

Para el estudio de la motivación de logro se empleó la escala de motivación de logro en contextos musicales elaborada por Zarza (2014). Este cuestionario consta de 26 preguntas que son evaluadas en una escala Likert de 9 puntos; en el cómputo general de la misma, arroja un estadístico de fiabilidad $\alpha$ de Cronbach de 0.775 . Muestra en su versión confirmatoria de 18 ítems ( $\alpha$ de Cronbach de 0.774) 5 subescalas diferenciadas en: interés con 4 ítems $(\square=$ 20), esfuerzo con 2 ítems ( $\square=10$ ), perfeccionismo con otros $2(\square=10)$ ítems, tarea con 8 ítems $(\square=40)$, y examen con $2(\square=10)$ ítems nuevamente.

En lo concerniente a las conductas de afrontamiento se plantearon dos preguntas, una relacionada el consumo de sustancias, medicamentos, tisanas y/o drogas de diverso tipo, y otra cuestión en la que se preguntaba si alguna vez se había querido abandonar los estudios musicales debido a problemas con la ansiedad escénica o la falta de motivación para llevarlos a cabo.

Relativo al análisis de datos, con el programa informático SPSS en su versión 19.0, se han llevado a cabo análisis descriptivos y correlacionales.

\section{Resultados}

Los factores de interés, esfuerzo, tarea y exámenes arrojan valores medios superiores a la media teórica del instrumento de evaluación de la motivación (Tabla 1). Estas medias, además, son significativamente diferentes a las medias teóricas en todos ellos. Se puede observar que en el factor interés $\mathrm{t}=38.622 ; \mathrm{p}=.000$; en el factor esfuerzo $\mathrm{t}=20.103 ; \mathrm{p}=.000$; para el factor exámenes $\mathrm{t}=10.301 ; \mathrm{p}=.000$; y para el factor tarea $\mathrm{t}=6.120 ; \mathrm{p}=.000$. Por su parte, el factor de perfeccionismo presenta una media significativamente inferior a la media teórica del instrumento $(\mathrm{t}=-5.632 ; \mathrm{p}=.001)$.

Tabla 1. Estadísticos descriptivos.

\begin{tabular}{|c|c|c|c|c|c|c|}
\hline & $\mathrm{N}$ & Mínimo & Máximo & Media & $\begin{array}{c}\text { Media } \\
\text { Teórica }\end{array}$ & Desv. típ. \\
\hline Interés & 154 & 20.00 & 36.00 & 31.9282 & 20 & 3.83265 \\
Esfuerzo & 154 & 4.00 & 18.00 & 14.5273 & 10 & 2.79479 \\
Perfeccionismo & 154 & 2.00 & 18.00 & 7.9935 & 10 & 4.42142 \\
Tarea & 154 & 21.00 & 64.00 & 45.0448 & 40 & 10.22994 \\
Exámenes & 154 & 5.00 & 18.00 & 12.2819 & 10 & 2.74911 \\
N válido (según lista) & 140 & & & & & \\
\hline
\end{tabular}

Analizada en términos porcentuales la población en función de su alejamiento de la media obtenida en desviaciones típicas, se aprecia que aproximadamente el $18.2 \%$ de los estudiantes muestran puntuaciones alejadas por la cola inferior en más de una desviación típica en el factor interés; este 
porcentaje se sitúa en torno al 13\% para el factor esfuerzo, en un $19.5 \%$ para el factor perfeccionismo, llega hasta aproximadamente un $18.8 \%$ para el factor tarea y se sitúa en un $17 \%$ para el factor exámenes.

De manera correlacional (Tabla 2), por una parte se observa que el interés se asocia significativa y directamente proporcional con los factores de esfuerzo $(\mathrm{r}=.588 ; \mathrm{p}=.000)$ y exámenes $(\mathrm{r}=.184 ; \mathrm{p}=.022)$ mientras que lo hace de manera inversa con el factor de perfeccionismo $(r=-.204 ; \mathrm{p}=.011)$. De otra parte, se ve cómo el factor tarea correlaciona a su vez te manera directamente proporcional y significativa con el factor de exámenes $(r=.467$; $\mathrm{p}=.000)$.

Tabla 2. Correlaciones.

\begin{tabular}{|c|c|c|c|c|c|}
\hline & Interés & Esfuerzo & Perfeccionismo & Tarea & Exámenes \\
\hline Interés & 1 & $.588^{* * *}$ & $-.204^{*}$ & & $.184^{* *}$ \\
\hline Esfuerzo & $.588^{* *}$ & 1 & & & \\
\hline Perfeccionismo & $-.204^{*}$ & & 1 & & \\
\hline Tarea & & & & 1 & $.467^{* *}$ \\
\hline
\end{tabular}

En lo concerniente al estudio de las conductas de afrontamiento, consumo de sustancias o pensamientos de abandono, se aprecia cómo en el caso del consumo de sustancias, el $33.8 \%$ de la población reconoce haber consumido algún tipo de sustancia para afrontar los requerimientos específicos de los conciertos públicos durante su periodo formativo. En este sentido, se ve cómo el $41.7 \%$ de las personas que han tomado algún tipo de sustancia han recurrido al empleo de fármacos, el $29.2 \%$ a productos de herbolario como tisanas o infusiones, un $16.7 \%$ combinaba bien productos de herbolario con medicamentos, y un $12.5 \%$ recurría al empleo de drogas o al consumo de alcohol.

En el caso de las conductas de abandono de los estudios musicales, de los 117 casos válidos, el $12 \%$ reconoce haber tenido pensamientos de abandono de los estudios mientras que un $88 \%$ indica que no ha querido abandonar los estudios musicales. En este sentido, se encuentra una asociación significativa entre las personas que han consumido sustancias y que han querido abandonar los estudios musicales $\left(\chi^{2}=4.184 ; p=.041\right)$.

Analizadas las posibles diferencias de medias que se pudieran producir en los factores motivacionales en función de las estrategias de afrontamiento, se observa que se producen diferencias de medias significativas en el factor tarea $(\mathrm{F}=4.402 ; \mathrm{p}=.038)$; siendo los estudiantes que no han consumido sustancias los que presentan una media superior $(46.2657$; D.T. $=9.03)$ a la de los estudiantes que sí que han consumido algún tipo de sustancia (43.4533; D.T. $=11.16$ ).

En el caso de considerar los pensamientos de abandono como factor fijo, se comprueba que también se producen diferencias significativas en las puntuaciones en función de una $\mathrm{u}$ otra respuesta. Así en el factor tarea $(\mathrm{F}=$ 
15.827; $\mathrm{p}=.000)$ las personas que no han tenido pensamientos de abandono presentan una media mayor $(46.6764$; D.T. $=9.38)$ que las personas que sí que han pensado en abandonar $(36.1429 ;$ D.T. $=8.55)$. De manera análoga ocurre con el factor exámenes $(\mathrm{F}=9.463 ; \mathrm{p}=.003)$ en donde las personas que no han presentado pensamientos de abandono tienen una media mayor (12.5020; D.T. $=2.55)$ que las personas que sí que han presentado pensamientos de abandono $(10.2143$; D.T. $=3.01)$.

\section{Conclusión}

El constructo de la motivación, entendido éste de manera multifactorial es uno de los constructos más importantes de cara al sostenimiento de una formación musical exitosa. En este sentido, se ha podido comprobar, al igual que en Zarza (2014) cómo los bajos niveles motivacionales están relacionados con la toma de sustancias. Del mismo modo, se ha podido constatar cómo la toma de sustancias junto con los bajos niveles motivacionales están relacionados con los pensamientos de abandono. Este tipo de conductas de consumo de sustancias, especialmente las que implican la toma de medicamentos o drogas, conllevan a largo plazo la habituación a las mismas sin que se reporten mejorías ni en los pensamientos de abandono ni en los niveles motivacionales, ocasionando en el largo plazo conductas disfuncionales (Rojo et al., 2009). De esta forma, se considera, tal y como se pone de manifiesto también en Zarza, Casanova y Orejudo (2016a, 2016b), que una de las vías para mejorar este tipo de conductas claramente desadaptativas debe partir desde el punto de vista pedagógico. Es decir, podría mejorar con la creación y asentamiento de nuevas directrices pedagógicas, tanto a nivel institucional como individual; unas directrices en las que se preste una especial atención de carácter individualizado al alumnado y en las que se pretenda también la mejora en las habilidades formativas de carácter técnicomusical del mismo de manera progresiva, fomentando un mayor control sobre la tarea específica de la ejecución musical.

Con todo ello seguro que se podrán reducir y modificar este tipo de conductas desadaptativas en conductas y cogniciones adaptativas en el conjunto del alumnado $\mathrm{y}$, por consiguiente, mejoraría notablemente la formación musical de los estudiantes.

\section{Referencias:}

1. David, N., Newen, A., \& Vogeley K. (2008). The "sense of agency" and its underlying cognitive and neural mechanisms. Consciousness and Cognition, 17, 523-534.

2. Gallagher, S. (2000). Philosophical conceptions of the self: implications for cognitive science. Trends in Cognitive Science, 4, 14-21. 
3. Gallagher, S. (2008). Direct perception in the intersubjective context. Consciousness and Cognition, 17, 535-543.

4. Manassero, M. A. \& Vázquez, A. (1998). Validación de una escala de motivación de logro. Psicothema, 10(2), 333-351.

5. Martin, A. J. (2001). The Student Motivation Scale: A tool for measuring and enhancing motivation. Australian Journal of Guidance and Counselling, 11, 1-20.

6. Martin, A. J. (2003). The Student Motivation Scale: Further testing of an instrument that measures school students' motivation. Australian Journal of Education, 47, 88-106.

7. Martin, A. J. (2007). Examining a multidimensional model of student motivation and engagement using a construct validation approach. British Journal of Educational Psychology, 77, 413-440.

8. Martin, A. J. (2008). How domain specific is motivation and engagement across school, sport, and music? A substantive-methodological synergy assessing young sportspeople and musicians. Contemporary Educational Psychology, 33, 785-813.

9. Renwick, J. M. \& McPherson, G. E. (2002). Interest and choice: Studentselected repertoire and its effect on practising behaviour. British Journal of Music Education, 19, 173-188.

10. Rojo, G., Pedrero, E.J., Ruiz, J.M., Llanero, M., Olivar, A., \& Puerta, C. (2009). Terapia ocupacional en la rehabilitación de la disfunción ejecutiva en adictos a sustancias. Trastornos Adictivos, 11(2), 96-105.

11. Weiner, B. (1986). An Attributional Theory of Motivation and Emotion. New York, NY: Springer-Verlag.

12. Zarza, F. J. (2014). Variables psicológicas y pedagógicas como predictoras de la ansiedad escénica en estudiantes de Grado Superior de Música de España. Tesis doctoral. Zaragoza: Universidad de Zaragoza.

13. Zarza, F. J., Casanova, O., \& Orejudo, S. (2016a). Ansiedad escénica y constructos psicológicos relacionados. Estudiantes de cinco conservatorios superiores de música españoles. RIEM. Revista Internacional de Educación Musical, 4, 13-24. doi:10.12967/RIEM2016-4-p013-024

14. Zarza, F. J., Casanova, O., \& Orejudo, S. (2016b). Estudios de música en los conservatorios superiores y ansiedad escénica en España. RECIEM. Revista Electrónica Complutense de Investigación en Educación Musical, 13, 50-63. doi:10.5209/RECIEM.49442 